\title{
ON THE ANALYSIS OF DEEP DRAWDOWNS FOR THE LÉVY INSURANCE RISK MODEL
}

\author{
DAVID LANDRIAULT, BIN LI, AND MOHAMED AMINE LKABOUS
}

\begin{abstract}
In this paper, we study the magnitude and the duration of deep drawdowns for the Lévy insurance risk model through the characterization of the Laplace transform of a related stopping time. Relying on a temporal approximation approach (e.g., Li et al. [17]), the proposed methodology allows for a unified treatment of processes with bounded and unbounded variation paths whereas these two cases used to be treated separately (e.g., Yin and Yuen [30]). In particular, we extend the results of Landriault et al. [14] and Surya [28]. We later analyze certain limiting cases of our main results where consistency with some known drawdown results in the literature will be shown.
\end{abstract}

\section{INTRODUCTION}

In insurance mathematics, drawdowns have drawn accrued interest in recent years. Drawdowns are well known to quantify negative fluctuations in an index value relative to its running maximum, a fact of utmost importance for risk management purposes. This is particularly true in insurance contexts, where the insurer is concerned with extreme downward movements in surplus levels. Over the past few decades, several risk measures involving drawdowns have been studied. For example, in the fund management industry, drawdown-based performance measures such as the Calmar, Burke and Martin ratios, are frequently used (e.g., Schuhmacher and Elin [26] and Bacon [5]). In finance and risk management, a great deal of research has been carried on reducing drawdown risks including but not limited to Grossman and Zhou [12] on portfolio optimization with drawdown constraints in a Black-Scholes framework, Cvitanic and Karatzas [9] on its generalization with several risky assets, and Chen et al. [8] on minimizing the probability that a drawdown occurs over a lifetime investment. Also, new portfolio sensitivities with respect to the running maximum and drawdowns of the underlying asset are defined by Pospisil and Vecer [24]. Shepp and Shiryaev [27] studied the pricing of the perpetual American options, also known as Russian options, where strong ties are shown to drawdown.

From a more theoretical standpoint, Taylor [29] studied the joint Laplace transform of the time to a given drawdown and its related running maximum for the class of Brownian motions. The generalization to a time-homogeneous diffusion process can be found in Lehoczky [16]. A more general treatment of drawdown quantities the Lévy insurance risk model has been studied by Mijatovic et al. [22] and Baurdoux et al. [6]. Building on the aforementioned papers, Palmowski and Tumilewicz [23] studied fair valuation of insurance contracts against drawdown (and drawup) of log-returns of stock price modelled by a spectrally negative geometric Lévy process. Also, Avram et al. [3] studied the pricing of Russian options for the same model.

Date: May 12, 2021.

Key words and phrases. Drawdown process, Drawdown duration, Lévy insurance risk processes. 
Another risk which aims to capture drops in capital levels is the so-called time under water, i.e. the duration from a previous running maximum to a new one. This duration, also known as the "Time to Recover" in the fund management industry, has been studied via an analysis of its Laplace transform by Landriault et al. [14] for general Lévy processes. The duration of drawdowns is also related to the idea of Parisian ruin with deterministic delays. The latter was first studied by Dassios and Wu [10,11] for the Brownian motion process and CramérLundberg process with exponential claims, respectively. In a more general setup, Loeffen et al. [20] generalized these results to the general class of spectrally negative Lévy processes. Also, for this type of ruin, an expression for the probability of Parisian ruin for the refracted process was studied by Lkabous et al. [18].

Recently, under the spectrally negative Lévy framework, Surya [28] used the spatial approximation approach (e.g., Loeffen et al. [20] and Landriault et al. [15]) to study the Laplace transform of the duration of drawdowns when the underlying process is monitored continuously, and the clock rings the first time the duration of a drawdown of a given magnitude exceeds a pre-specified time threshold $r>0$. It is worth pointing out that, in [14] and [28], the analysis of the Laplace transform of the duration of a drawdown is splitted into two cases, depending on whether the process is of bounded or unbounded variation.

From a risk management standpoint, large drawdowns should be considered as extreme events of which both the severity and duration need to be given special consideration. In this paper, we consider a particular stopping time related to the underlying drawdown process which aims to capture both the severity and duration aspects. We make use of the Poisson observation scheme to conduct the technical analysis (e.g., [1], [2] and [17]). We consider the following observation scheme for monitoring the risk process:

- in shallow-water drawdown-zone (when the drawdown process $Y$ does not exceed a pre-specified level $a$ ), the drawdown process is observed at the arrival times of an independent Poisson process;

- in deep-water drawdown-zone (when the drawdown process $Y$ exceeds level $a$ ), the drawdown process is observed continuously until either the drawdown process goes above level $b$ or a pre-specified time threshold $r$ is reached before the process returns in the shallow-water drawdown-zone.

Note that the barrier $b$ can be interpreted as a critical depth level above which the process is unlikely to recover from its running maximum whereas the level $a$ could be seen as a recovery barrier. The time threshold $r$ can also be interpreted as a grace period given to the drawdown process to return into the shallow-water drawdown zone.

An important contribution of this paper is that the Poissonian observation scheme provides a unified methodology to carry the analysis for processes with bounded or unbounded variation paths, whereas these two cases are treated separately using the traditional spatial approximation approach (see, e.g., Albrecher et al. [2] and Li et al. [17]). We later consider limiting cases of our main results in order to recover known results pertaining to the drawdown process in the literature. Our model can also be applied in modelling the ruin of a dividend-paying company as in Avram et al. [4], Pérez and Yamazaki [25] among others.

The rest of the paper is organized as follows. In Section 2, we give the necessary background material on spectrally negative Lévy processes and scale functions. In Section 3, we present our model in more details together with some fluctuation identities for the drawdown process. The main result as well as a (new) technical lemma are presented in Section 4 . In Section 5 , we analyze limiting cases in order to recover previous drawdown-related results. Many proofs are postponed to the Appendices $\mathrm{A}$ and $\mathrm{B}$. 


\section{Preliminaries}

In this section, we present the necessary background material on spectrally negative Lévy processes.

A Lévy insurance risk process $X=\left\{X_{t}\right\}_{t>0}$ is a process with stationary and independent increments and no positive jumps. To avoid trivialities, we exclude the case where $X$ has monotone paths. As the Lévy process $X$ has no positive jumps, its Laplace transform exists, namely

$$
\mathbb{E}\left[\mathrm{e}^{\lambda X_{t}}\right]=\mathrm{e}^{t \psi(\lambda)}
$$

for all $\lambda, t \geq 0$, where

$$
\psi(\lambda)=\gamma \lambda+\frac{1}{2} \sigma^{2} \lambda^{2}+\int_{-\infty}^{0}\left(\mathrm{e}^{\lambda z}-1-\lambda z \mathbf{1}_{\{z>-1\}}\right) \Pi(\mathrm{d} z),
$$

for $\gamma \in \mathbb{R}$ and $\sigma \geq 0$, and where $\Pi$ is a $\sigma$-finite measure on $(-\infty, 0)$ called the Lévy measure of $X$ which is assumed to satisfy

$$
\int_{-\infty}^{0}\left(1 \wedge z^{2}\right) \Pi(\mathrm{d} z)<\infty
$$

Throughout, we will use the standard Markovian notation: the law of $X$ when starting from $X_{0}=x$ is denoted by $\mathbb{P}_{x}$ and the corresponding expectation by $\mathbb{E}_{x}$. For simplicity, we write $\mathbb{P}$ and $\mathbb{E}$ when $x=0$. We also use the following notation: the law of $X$ when starting from $X_{0}=x$ and with current maximum $y(\geq x)$ is denoted by $\mathbb{P}_{x}^{y}$ and the corresponding expectation by $\mathbb{E}_{x}^{y}$. We write $\mathbb{P}^{y}$ and $\mathbb{E}^{y}$ when $x=0$. We also denote the first passage time above $b$ of $X$ by

$$
\tau_{b}^{+}=\inf \left\{t \geq 0: X_{t} \geq b\right\}
$$

We now present the definition of the scale functions $W_{q}$ and $Z_{q}$ of $X$. First, recall that there exists a function $\Phi:[0, \infty) \rightarrow[0, \infty)$ defined by $\Phi_{q}=\sup \{\lambda \geq 0 \mid \psi(\lambda)=q\}$ (the right-inverse of $\psi$ ) such that

$$
\psi\left(\Phi_{q}\right)=q, \quad q \geq 0 .
$$

Now, for $q \geq 0$, the $q$-scale function for the process $X$, namely $W_{q}(x)(x \geq 0)$, is defined as the continuous function on $[0, \infty)$ with Laplace transform

$$
\int_{0}^{\infty} \mathrm{e}^{-\lambda y} W_{q}(y) \mathrm{d} y=\frac{1}{\psi_{q}(\lambda)}, \quad \lambda>\Phi_{q},
$$

where $\psi_{q}(\lambda)=\psi(\lambda)-q$. This function is unique, positive and strictly increasing for $x \geq 0$ and is further continuous for $q \geq 0$. For convenience, we extend $W_{q}$ to the whole real line by setting $W_{q}(x)=0$ for $x<0$. We write $W=W_{0}$ when $q=0$.

It is well known that the scale function $W_{q}$ belongs to $C^{1}(0, \infty)$ if the process $X$ has paths of unbounded variation or if the process $X$ has paths of bounded variation and the Lévy measure has no atom.

The initial value of $W_{q}$ is given by

$$
W_{q}(0+)= \begin{cases}1 / c & \text { when } \mathrm{X} \text { has bounded variation, } \\ 0 & \text { when } \mathrm{X} \text { has unbounded variation }\end{cases}
$$


where $c=\gamma+\int_{(-1,0)}|z| \Pi(\mathrm{d} z)$.

We also define another scale function $Z_{q}(x, \theta)$ by

$$
Z_{q}(x, \theta)=\mathrm{e}^{\theta x}\left(1-\psi_{q}(\theta) \int_{0}^{x} \mathrm{e}^{-\theta y} W_{q}(y) \mathrm{d} y\right), \quad x \geq 0,
$$

and $Z_{q}(x, \theta)=\mathrm{e}^{\theta x}$ for $x<0$. For $\theta=0$, we get

$$
Z_{q}(x, 0)=Z_{q}(x)=1+q \int_{0}^{x} W_{q}(y) \mathrm{d} y, \quad x \in \mathbb{R} .
$$

Using (1), whenever $\theta>\Phi_{q}$, an alternative representation for $Z_{q}(x, \theta)$ is given by

$$
Z_{q}(x, \theta)=\psi_{q}(\theta) \int_{0}^{\infty} \mathrm{e}^{-\theta y} W_{q}(x+y) \mathrm{d} y, \quad x \geq 0,
$$

We denote the derivative of $Z_{q}(x, \theta)$ with respect to $x$ by

$$
Z_{q}^{\prime}(x, \theta)=\theta Z_{q}(x, \theta)-\psi_{q}(\theta) W_{q}(x) .
$$

A second generation scale function was introduced by Loeffen et al. [21], that is, for $p, p+q \geq 0$ and $x \in \mathbb{R}$,

$$
\begin{aligned}
\mathcal{W}_{a}^{(p, q)}(x) & =W_{p}(x)+q \int_{a}^{x} W_{p+q}(x-y) W_{p}(y) \mathrm{d} y \\
& =W_{p+q}(x)-q \int_{0}^{a} W_{p+q}(x-y) W_{p}(y) \mathrm{d} y
\end{aligned}
$$

whose Laplace transform is of the form

$$
\int_{0}^{\infty} \mathrm{e}^{-\theta z} \mathcal{W}_{a}^{(p, q)}(a+z) \mathrm{d} z=\frac{Z_{p}(a, \theta)}{\psi_{p+q}(\theta)}, \quad \theta>\Phi_{p+q}
$$

Note that the two expressions on the right-hand side of $(7)$ can be shown to be equivalent using the following identity from Loeffen et al. [21]: for $p, q \geq 0$ and $x \in \mathbb{R}$,

$$
(p-q) \int_{0}^{x} W_{q}(x-y) W_{p}(y) \mathrm{d} y=W_{p}(x)-W_{q}(x) .
$$

The derivative of $\mathcal{W}_{a}^{(p, q)}$ with respect to $x$ is given by

$$
\mathcal{W}_{a}^{(p, q)^{\prime}}(x)=W_{p+q}^{\prime}(x)-q \int_{0}^{a} W_{p+q}^{\prime}(x-y) W_{p}(y) \mathrm{d} y .
$$

Also, fluctuation identities involving delays for spectrally negative Lévy processes are expressed in terms of new scale functions. In Loeffen et al. [19], the so-called delayed q-scale function of $X$ is introduced, that is, for $q \geq 0, r>0$ and $x \in \mathbb{R}$,

$$
\Lambda^{(q)}(x, r)=\int_{0}^{\infty} W_{q}(x+z) \frac{z}{r} \mathbb{P}\left(X_{r} \in \mathrm{d} z\right) .
$$

We write $\Lambda=\Lambda^{(0)}$ when $q=0$. For later use, it can be shown that

$$
\int_{0}^{\infty} \mathrm{e}^{-\theta r}\left(\mathrm{e}^{-q r} \Lambda^{(q)}(x, r)\right) \mathrm{d} r=\frac{Z_{q}\left(x, \Phi_{\theta+q}\right)}{\theta}, \quad \theta>0, x \in \mathbb{R},
$$

and consequently,

$$
\Lambda^{(q)}(0, r)=\mathrm{e}^{q r} .
$$


We also note that the partial derivative of $\Lambda^{(q)}$ with respect to $x$ is given by

$$
\Lambda^{(q)^{\prime}}(x, r)=\int_{0}^{\infty} W_{q}^{\prime}(x+z) \frac{z}{r} \mathbb{P}\left(X_{r} \in \mathrm{d} z\right) .
$$

The well-known expression for the Laplace transform of the first passage time above $a$ is given by

$$
\mathbb{E}_{x}\left[\mathrm{e}^{-q \tau_{a}^{+}}\right]=\mathrm{e}^{\Phi_{q}(x-a)} .
$$

Finally, we recall Kendall's identity that provides the distribution of the first upward crossing of a specific level (see $[7$, Corollary VII.3]): on $(0, \infty) \times(0, \infty)$, we have

$$
r \mathbb{P}\left(\tau_{z}^{+} \in \mathrm{d} r\right) \mathrm{d} z=z \mathbb{P}\left(X_{r} \in \mathrm{d} z\right) \mathrm{d} r .
$$

For more details on spectrally negative Lévy processes and fluctuation identities, we refer the reader to Kyprianou [13].

\section{OUR MODEL AND PROBlem FORMULATION}

The drawdown process of a Lévy insurance risk process $X$ is defined as

$$
Y_{t}=\bar{X}_{t} \vee y-X_{t}, \quad t \geq 0,
$$

where $\bar{X}_{t}=\sup _{s<t} X_{s}$ is the running maximum of $X$ up to time $t$ and $y$ is a past maximum achieved by $X$. The first passage times of $Y$ are given by

$$
\rho_{a}^{-}=\inf \left\{t \geq 0: Y_{t} \leq a\right\} \quad \text { and } \quad \rho_{b}^{+}=\inf \left\{t \geq 0: Y_{t} \geq b\right\},
$$

with the convention inf $\emptyset=\infty$. We define the following discrete observation time nodes $\left\{\xi_{n}\right\}_{n \in \mathbb{N}_{+}}$where $\xi_{0}=0$ and, for $n \in \mathbb{N}_{+}$,

$$
\xi_{n}=\xi_{n-1}+\rho_{a}^{-} \circ \theta_{\xi_{n-1}}+\mathrm{e}_{n}^{\lambda}, \quad \text { for } n \in \mathbb{N}_{+},
$$

where $\theta$ is the Markov shift operator $\left(Y_{s} \circ \theta_{t}=Y_{s+t}\right)$ and $\left\{\mathrm{e}_{n}^{\lambda}\right\}_{n \in \mathbb{N}_{+}}$is a sequence of independent and identically distributed (iid) exponential rv's with mean $1 / \lambda>0$ (also independent of $X$ ).

The duration of deep drawdowns with ultimate barrier $b$, a recovery barrier $a(<b)$ and a pre-specified time threshold $r>0$ is defined as

$$
\kappa_{a, b}^{\lambda, r}=\inf \left\{t \in\left(\xi_{n},\left(\rho_{a}^{-} \circ \theta_{\xi_{n}}\right)\right): Y_{t}>b \text { or } t-\xi_{n} \geq r, n \in \mathbb{N}\right\} .
$$

More specifically, when the drawdown process $Y$ is below a pre-specified level $a$, it is monitored discretely according to the arrival times of an independent Poisson process with rate $\lambda$. As soon as the drawdown process is observed above level $a$, the observation scheme reverts to the continuous scheme at which time a fixed period $r$ is granted for the drawdown process $Y$ to return to level $a$ contingent on the drawdown level never reaches the ultimate barrier level $b$ in the process (see Figure 1 for a simple path illustration). We point out that the following two stopping times can be viewed as limiting cases of (17)

$$
\kappa_{a}^{\lambda, r}=\inf \left\{t \in\left(\xi_{n},\left(\rho_{a}^{-} \circ \theta_{\xi_{n}}\right)\right): t-\xi_{n} \geq r, n \in \mathbb{N}\right\},
$$

and

$$
\kappa_{a}^{r}=\inf \left\{t>0: t-g_{t}^{a} \geq r\right\},
$$

where $g_{t}^{a}=\sup \left\{s \leq t: Y_{t} \leq a\right\}$ is the last time before time $t$ that the process $Y$ is below level $a>0$. When $a=0$, we write $\kappa_{0}^{r}=\kappa^{r}$. We point out that the stopping times $\kappa_{a}^{r}$ and $\kappa^{r}$ were studied in [14] and [28], respectively. 
Next, we recall some fluctuation identities for the drawdown process $Y$ which will be useful in the sequel. For $a \leq y \leq b$, we know from e.g. Proposition 2.5 of Surya [28], that

$$
\mathbb{E}^{y}\left[\mathrm{e}^{-q \rho_{a}^{-}} \mathbf{1}_{\left\{\rho_{a}^{-}<\rho_{b}^{+}\right\}}\right]=\frac{W_{q}(b-y)}{W_{q}(b-a)},
$$

and

$$
\mathbb{E}^{y}\left[\mathrm{e}^{-q \rho_{b}^{+}} \mathbf{1}_{\left\{\rho_{b}^{+}<\rho_{a}^{-}\right\}}\right]=Z_{q}(b-y)-\frac{W_{q}(b-y)}{W_{q}(b-a)} Z_{q}(b-a) .
$$

For convenience, we recall a result from Albrecher et al. [2] concerning the joint Laplace transform of $\left(T_{a}^{+}, Y_{T_{a}^{+}}\right)$, where

$$
T_{a}^{+}=\min \left\{\xi_{i}: Y_{\xi_{i}}>a, i \in \mathbb{N}\right\} .
$$

Lemma 1. For $s=y-x$ (where $y \geq x), a, \lambda>0$ and $p, q \geq 0$,

$$
\mathbb{E}_{x}^{y}\left[\mathrm{e}^{-q T_{a}^{+}-p\left(Y_{T_{a}^{+}}-a\right)}\right]=\frac{\lambda}{\lambda-\psi_{q}(p)}\left(Z_{q}(a-s, p)-\frac{Z_{q}\left(a-s, \Phi_{\lambda+q}\right)}{Z_{q}^{\prime}\left(a, \Phi_{\lambda+q}\right)} Z_{q}^{\prime}(a, p)\right) .
$$

Letting $\lambda \rightarrow \infty, 22$ converges to the following expression of the joint Laplace transform of $\rho_{a}^{+}$and the overshoot $Y_{\rho_{a}^{+}}$given in Theorem 1 of Avram et al. [3].

Lemma 2. For $s=y-x$ (where $y \geq x), a>0$ and $p, q \geq 0$,

$$
\mathbb{E}_{x}^{y}\left[\mathrm{e}^{-q \rho_{a}^{+}-p\left(Y_{\rho_{a}^{+}}-a\right)}\right]=Z_{q}(a-s, p)-\frac{W_{q}(a-s)}{W_{q}^{\prime}(a)} Z_{q}^{\prime}(a, p) .
$$

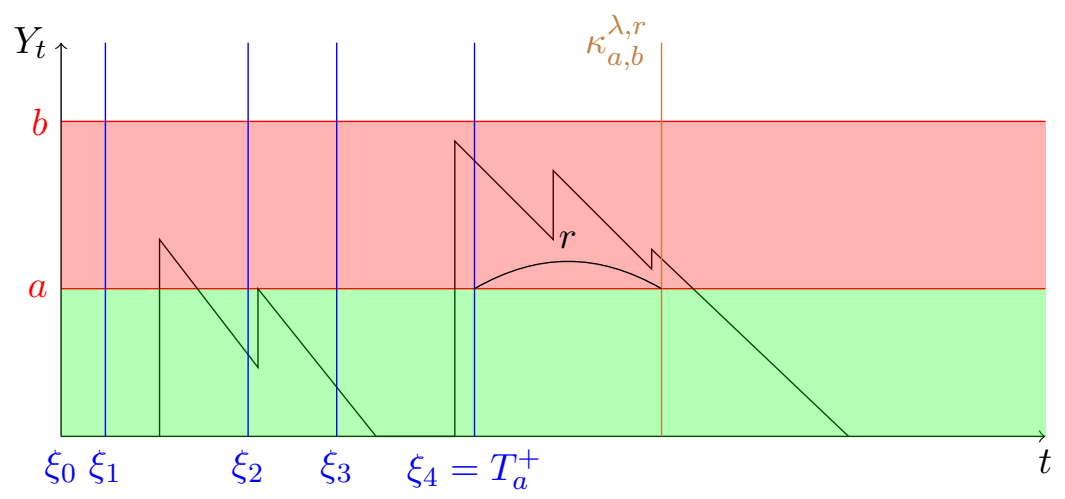

Figure 1. Illustration of the duration of drawdowns $\kappa_{a, b}^{\lambda, r}$. The red and green regions correspond to the continuous and discrete observation regions respectively.

\section{MAin Results}

In this section, we state in Theorem 6 our main result pertaining to the Laplace transform of $\kappa_{a, b}^{\lambda, r}$. The proof relies on a temporal argument approach similar to e.g., Li et al. [17. Before presenting our main result, we first give the following new results for the drawdown process $Y$. 
Lemma 3. For $s=y-x$ (where $y \geq x), a, z, \lambda>0$ and $p, q \geq 0$,

$$
\begin{aligned}
& \mathbb{E}_{x}^{y}\left[\mathrm{e}^{-q T_{a}^{+}} \mathbf{1}_{\left\{Y_{T_{a}^{+}}-a \in \mathrm{d} z, T_{a}^{+}<\infty\right\}}\right] \\
& =\lambda\left(\frac{Z_{q}\left(a-s, \Phi_{\lambda+q}\right)}{Z_{q}^{\prime}\left(a, \Phi_{\lambda+q}\right)}\left(\mathcal{W}_{a}^{(q, \lambda) \prime}(a+z)-\lambda W_{q}(a) W_{q+\lambda}(z)\right)-\mathcal{W}_{a-s}^{(q, \lambda)}(a-s+z)\right) \mathrm{d} z .
\end{aligned}
$$

Consequently,

$$
\begin{aligned}
& \mathbb{E}_{x}^{y}\left[\mathrm{e}^{-q T_{a}^{+}} W_{p}\left(b-Y_{T_{a}^{+}}\right) \mathbf{1}_{\left\{T_{a}^{+}<\infty\right\}}\right] \\
= & \lambda \frac{Z_{q}\left(a-s, \Phi_{\lambda+q}\right)}{Z_{q}^{\prime}\left(a, \Phi_{\lambda+q}\right)} \int_{0}^{b-a} W_{p}(b-a-z)\left(\mathcal{W}_{a}^{(q, \lambda) \prime}(a+z)-\lambda W_{q}(a) W_{q+\lambda}(z)\right) \mathrm{d} z \\
& -\lambda \int_{0}^{b-a} W_{p}(b-a-z) \mathcal{W}_{a-s}^{(q, \lambda)}(a-s+z) \mathrm{d} z,
\end{aligned}
$$

and

$$
\begin{aligned}
\mathbb{E}_{x}^{y}\left[\mathrm{e}^{-q T_{a}^{+}} Z_{p}\left(b-Y_{T_{a}^{+}}\right) \mathbf{1}_{\left\{T_{a}^{+}<\infty\right\}}\right] \\
=\lambda \frac{Z_{q}\left(a-s, \Phi_{\lambda+q}\right)}{Z_{q}^{\prime}\left(a, \Phi_{\lambda+q}\right)}\left(\int_{0}^{\infty} Z_{p}(b-a-z) \mathcal{W}_{a}^{(q, \lambda) \prime}(a+z)-\lambda W_{q}(a) W_{q+\lambda}(a)\right) \mathrm{d} z \\
\quad-\lambda \int_{0}^{\infty} Z_{p}(b-a-z) \mathcal{W}_{a-s}^{(q, \lambda)}(a-s+z) \mathrm{d} z .
\end{aligned}
$$

Proof. See the proof in Appendix A

Remark 4. As shown in [14], there exists a relationship between the potential measure

$\mathbb{P}_{x}^{y}\left(Y_{\mathrm{e}_{q}}-a \in \mathrm{d} z, T_{a}^{+}>\mathrm{e}_{q}\right)$ and the Gerber-Shiu function on the left-hand side of (24). This is the case as

$$
\begin{aligned}
\mathbb{E}_{x}^{y}\left[\mathrm{e}^{-q T_{a}^{+}} \mathbf{1}_{\left.\left\{Y_{T_{a}^{+}}-a \in \mathrm{d} z, T_{a}^{+}<\infty\right\}\right]}\right. & =\int_{0}^{\infty} \mathrm{e}^{-q t} \mathbb{P}_{x}^{y}\left(Y_{t}-a \in \mathrm{d} z, T_{a}^{+} \in \mathrm{d} t\right) \\
& =\lambda \int_{0}^{\infty} \mathrm{e}^{-q t} \mathbb{P}_{x}^{y}\left(Y_{t}-a \in \mathrm{d} z, T_{a}^{+}>t\right) \mathrm{d} t \\
& =\frac{\lambda}{q} \mathbb{P}_{x}^{y}\left(Y_{\mathrm{e}_{q}}-a \in \mathrm{d} z, T_{a}^{+}>\mathrm{e}_{q}\right) .
\end{aligned}
$$

Remark 5. An interesting and simple proof to obtain the continuous analogue of (25) is presented next. From (23), we have

$$
\frac{\mathbb{E}_{x}^{y}\left[\mathrm{e}^{-q \rho_{a}^{+}-p\left(Y_{\rho_{a}^{+}}-a\right)}\right]}{\psi_{q}(p)}=\frac{Z_{q}(a-s, p)}{\psi_{q}(p)}-\frac{W_{q}(a-s)}{W_{q}^{\prime}(a)} \frac{Z_{q}^{\prime}(a, p)}{\psi_{q}(p)},
$$

and using (1), it follows that

$$
\begin{aligned}
& \frac{\mathbb{E}_{x}^{y}\left[\mathrm{e}^{-q \rho_{a}^{+}-p\left(Y_{\rho_{a}^{+}}-a\right)}\right]}{\psi_{q}(p)}=\int_{0}^{\infty} \mathrm{e}^{-p z}\left(\int_{0}^{z} W_{q}(z-y) \mathbb{E}_{x}^{y}\left[\mathrm{e}^{-q \rho_{a}^{+}} \mathbf{1}_{\left\{Y_{\rho_{a}^{+}}-a \in \mathrm{d} y, \rho_{a}^{+}<\infty\right\}}\right]\right) \mathrm{d} z \\
& =\int_{0}^{\infty} \mathrm{e}^{-p z} \mathbb{E}_{x}^{y}\left[\mathrm{e}^{-q \rho_{a}^{+}} W_{q}\left(z+a-Y_{\rho_{a}^{+}}\right) \mathbf{1}_{\left\{\rho_{a}^{+}<\infty\right\}}\right] \mathrm{d} z .
\end{aligned}
$$


Thus, by making use of (1), (37) and (39) and by uniqueness of Laplace transforms, we obtain

$$
\mathbb{E}_{x}^{y}\left[\mathrm{e}^{-q \rho_{a}^{+}} W_{q}\left(z-a-Y_{\rho_{a}^{+}}\right) \mathbf{1}_{\left\{\rho_{a}^{+}<\infty\right\}}\right]=W_{q}(a-s+z)-\frac{W_{q}(a-s)}{W_{q}^{\prime}(a)} W_{q}^{\prime}(a+z) .
$$

For notational convenience, we first introduce two auxiliary functions, namely $g_{\lambda}^{(q)}(a, b, r, y)$ and $h_{\lambda}^{(q)}(a, b, r, y)$, defined as

$$
\begin{aligned}
& g_{\lambda}^{(q)}(a, b, r, y)=\mathbb{E}^{y}\left[\mathrm{e}^{-q T_{a}^{+}} \mathbb{E}^{Y_{a}^{+}}\left[\mathrm{e}^{-q \rho_{a}^{-}} \mathbf{1}_{\left\{\rho_{a}^{-} \leq r \wedge \rho_{b}^{+}\right\}}\right] \mathbf{1}_{\left\{T_{a}^{+}<\infty\right\}}\right], \\
& h_{\lambda}^{(q)}(a, b, r, y)=\mathbb{E}^{y}\left[\mathrm{e}^{-q T_{a}^{+}} \mathbb{E}^{Y_{a}^{+}}\left[\mathrm{e}^{-q\left(r \wedge \rho_{b}^{+}\right)} \mathbf{1}_{\left\{\rho_{a}^{-}>r \wedge \rho_{b}^{+}\right\}}\right] \mathbf{1}_{\left\{T_{a}^{+}<\infty\right\}}\right],
\end{aligned}
$$

for $a, y \in \mathbb{R}, q, \theta \geq 0, a, r, \lambda>0$, and $b>a$. The functions $g_{\lambda}^{(q)}$ and $h_{\lambda}^{(q)}$ are closely related to Lemma 3 and they are defined trough their LT with respect to $r$. Thus, for a given scale function, it could be possible to compute the LT and take inverse analytically (or numerically) with respect to $r$. This can be seen by the Laplace transforms of these two functions in (45) and (46), respectively. $\kappa_{a, b}^{\lambda, r}$

In the next theorem, we provide a semi-explicit expression for the Laplace transform of

Theorem 6. For $v=y-x$ where $y \geq x, a, b, q \geq 0$ and $r, \lambda>0$,

$$
\mathbb{E}_{x}^{y}\left[\mathrm{e}^{-q\left(\kappa_{a, b}^{\lambda, r}-r\right)}\right]=h_{\lambda}^{(q)}(a, b, r, v)+\frac{g_{\lambda}^{(q)}(a, b, r, v)}{1-g_{\lambda}^{(q)}(a, b, r, a)} h_{\lambda}^{(q)}(a, b, r, a) .
$$

Proof. See proof in Appendix A.

Now, we turn our attention to the Laplace transform of $\kappa_{a}^{\lambda, r}$ which can be derived as a limiting case of $\kappa_{a, b}^{\lambda, r}$ when $b \rightarrow \infty$. Again, for notational convenience, we define the following auxiliary functions, for $\lambda, r>0, q \geq 0$ and $u \in \mathbb{R}$,

$$
\begin{aligned}
\mathcal{D}^{(q)}(u, r) & =Z_{q}(u)+q \int_{0}^{r} \Lambda^{(q)}(u, s) \mathrm{d} s \\
\mathcal{K}^{(q)}(u, r, \lambda) & =\mathrm{e}^{\lambda r}\left(Z_{q}\left(u, \Phi_{\lambda+q}\right)-\lambda \int_{0}^{r} \mathrm{e}^{-(\lambda+q) s} \Lambda^{(q)}(u, s) \mathrm{d} s\right),
\end{aligned}
$$

and we denote their respective partial derivatives with respect to $u$ by

$$
\begin{aligned}
\mathcal{D}^{(q)^{\prime}}(u, r) & =\frac{\partial}{\partial u} \mathcal{D}^{(q)}(u, r)=q W_{q}(u)+q \int_{0}^{r} \Lambda^{(q)^{\prime}}(u, s) \mathrm{d} s, \\
\mathcal{K}^{(q)^{\prime}}(u, r, \lambda) & =\frac{\partial}{\partial u} \mathcal{K}^{(q)}(u, r, \lambda)=\mathrm{e}^{\lambda r}\left(Z_{q}^{\prime}\left(u, \Phi_{\lambda+q}\right)-\lambda \int_{0}^{r} \mathrm{e}^{-(\lambda+q) s} \Lambda^{(q)^{\prime}}(u, s) \mathrm{d} s\right) .
\end{aligned}
$$

Corollary 7. For $v=y-x$ where $y \geq x, q \geq 0$ and $a, r, \lambda>0$,

$$
\mathbb{E}_{x}^{y}\left[\mathrm{e}^{-q\left(\kappa_{a}^{\lambda, r}-r\right)}\right]=\frac{\lambda}{\lambda+q}\left(\mathcal{D}^{(q)}(a-v, r)-\frac{\mathcal{K}^{(q)}(a-v, r, \lambda)}{\mathcal{K}^{(q)^{\prime}}(a, r, \lambda)} \mathcal{D}^{(q) \prime}(a, r)\right) .
$$

Proof. See proof in Appendix A.

Setting $q=0$ in (6), the following corollary is an immediate consequence of the last corollary. 
Corollary 8. For $y \geq x$ and $a, r, \lambda>0$,

$$
\mathbb{P}_{x}^{y}\left(\kappa_{a}^{\lambda, r}<\infty\right)=1
$$

\section{Limiting ANALYSiS}

In this section, we consider certain limiting cases of Theorem 6 and show their consistency with known results in the literature.

We begin by examining the limiting case of Corollary 7 when $\lambda \rightarrow \infty$. We later show that this result is in agreement with Theorem 1 in Surya 28.

Proposition 9. For $v=y-x$ where $y \geq x, q \geq 0$ and $a, r>0$, we have

$$
\lim _{\lambda \rightarrow \infty} \mathbb{E}_{x}^{y}\left[\mathrm{e}^{-q\left(\kappa_{a}^{\lambda, r}-r\right)}\right]=D^{(q)}(a-v, r)-\frac{\Lambda^{(q)}(a-v, r)}{\Lambda^{(q) \prime}(a, r)} D^{(q) \prime}(a, r) .
$$

Proof. First, we show a limiting result pertaining to the function $\mathcal{K}^{(q)}$ defined in 30 , namely

$$
\lim _{\lambda \rightarrow \infty} \mathcal{K}^{(q)}(u, r, \lambda)=\mathrm{e}^{-q r} \Lambda^{(q)}(u, r) .
$$

Using (5) and (11), we rewrite 30 as

$$
\mathcal{K}^{(q)}(u, r, \lambda)=\lambda \mathrm{e}^{\lambda r} \int_{0}^{\infty} W_{q}(u+z)\left(\mathrm{e}^{-\Phi_{\lambda+q} z}-\int_{0}^{r} \mathrm{e}^{-(\lambda+q) s} \mathbb{P}\left(\tau_{z}^{+} \in \mathrm{d} s\right)\right) \mathrm{d} z
$$

Later, with the help of (14) and Kendall's identity in (15), it becomes

$$
\begin{aligned}
\mathcal{K}^{(q)}(u, r, \lambda) & =\int_{0}^{\infty} W_{q}(u+z)\left(\lambda \mathrm{e}^{\lambda r} \int_{r}^{\infty} \mathrm{e}^{-(\lambda+q) s} \mathbb{P}\left(\tau_{z}^{+} \in \mathrm{d} s\right)\right) \mathrm{d} z \\
& =\lambda \int_{r}^{\infty} \mathrm{e}^{-\lambda(s-r)}\left(\mathrm{e}^{-q s} \int_{0}^{\infty} W_{q}(u+z) \frac{z}{s} \mathbb{P}\left(X_{s} \in \mathrm{d} z\right)\right) \mathrm{d} s .
\end{aligned}
$$

By taking the limit as $\lambda \rightarrow \infty$ on both sides of (34) and using the initial value theorem of Laplace transform,

$$
\begin{aligned}
\lim _{\lambda \rightarrow \infty} \mathcal{K}^{(q)}(u, r, \lambda) & =\lim _{\lambda \rightarrow \infty} \lambda \int_{r}^{\infty} \mathrm{e}^{-\lambda(s-r)}\left(\mathrm{e}^{-q s} \int_{0}^{\infty} W_{q}(u+z) \frac{z}{s} \mathbb{P}\left(X_{s} \in \mathrm{d} z\right)\right) \mathrm{d} s \\
& =\mathrm{e}^{-q r} \Lambda^{(q)}(u, r) .
\end{aligned}
$$

Finally, using similar steps, one can easily show that

$$
\lim _{\lambda \rightarrow \infty} \mathcal{K}^{(q)^{\prime}}(u, r, \lambda)=\mathrm{e}^{-q r} \Lambda^{(q)^{\prime}}(u, r) .
$$

This ends the proof.

Remark 10. We point out that, by substituting the expressions of $\mathcal{D}^{(q)}, \Lambda^{(q)}, \mathcal{D}^{(q)^{\prime}}$ and $\Lambda^{(q)^{\prime}}$ in (33), Proposition 9 corresponds to Theorem 1 of Surya [28] which has been presented as

$$
\begin{aligned}
\mathbb{E}_{x}^{y}\left[\mathrm{e}^{\left.-q\left(\kappa_{a}^{r}-r\right)\right]=}\right. & 1+q \int_{0}^{a-v} W_{q}(u) \mathrm{d} u-q \frac{\Lambda^{(q)}(a-v, r)}{\Lambda^{(q) \prime}(a, r)} W_{q}(a) \\
& +q \int_{0}^{r}\left(\Lambda^{(q)}(a-v, t)-\frac{\Lambda^{(q)}(a-v, r)}{\Lambda^{(q) \prime}(a, r)} \Lambda^{(q) \prime}(a, t)\right) \mathrm{d} t .
\end{aligned}
$$


Now, in order to recover the Laplace transform of $T_{a}^{+}$in $(22)$ (for $p=0$ ), we state (without proof) the limiting case when $r \rightarrow 0$.

Proposition 11. For $s=y-x$ where $y \geq x, q \geq 0$ and $a, r>0$,

$$
\lim _{r \rightarrow 0} \mathbb{E}_{x}^{y}\left[\mathrm{e}^{-q\left(\kappa_{a}^{\lambda, r}-r\right)}\right]=\frac{\lambda}{\lambda+q}\left(Z_{q}(a-s)-q \frac{Z_{q}\left(a-s, \Phi_{\lambda+q}\right)}{Z_{q}^{\prime}\left(a, \Phi_{\lambda+q}\right)} W_{q}(a)\right) .
$$

Combining the last two propositions, we therefore have

$$
\lim _{r \rightarrow 0} \lim _{\lambda \rightarrow \infty} \mathbb{E}_{x}^{y}\left[\mathrm{e}^{-q\left(\kappa_{a}^{\lambda, r}-r\right)}\right]=\lim _{\lambda \rightarrow \infty} \lim _{r \rightarrow 0} \mathbb{E}_{x}^{y}\left[\mathrm{e}^{-q\left(\kappa_{a}^{\lambda, r}-r\right)}\right]=\mathbb{E}_{x}^{y}\left[\mathrm{e}^{-q \rho_{a}^{+}}\right]
$$

\section{ACKNOWLEDGEMENTS}

Support from grants from the Natural Sciences and Engineering Research Council of Canada is gratefully acknowledged by David Landriault and Bin Li (grant numbers 341316 and 05828, respectively). Support from the Canada Research Chair Program is gratefully acknowledged by David Landriault.

\section{REFERENCES}

[1] H. Albrecher and J. Ivanovs, Strikingly simple identities relating exit problems for Lévy processes under continuous and Poisson observations, Stochastic Process. Appl. 127 (2017), no. 2, 643-656.

[2] H. Albrecher, J. Ivanovs, and X. Zhou, Exit identities for Lévy processes observed at Poisson arrival times, Bernoulli 22 (2016), no. 3, 1364-1382.

[3] F Avram, A E Kyprianou, and M R Pistorius, Exit problems for spectrally negative Lévy processes and applications to (Canadized) Russian options, Ann. Appl. Probab. 14 (2004), no. 1, 215.

[4] F. Avram, J.-L. Pérez, and K. Yamazaki, Spectrally negative Lévy processes with parisian reflection below and classical reflection above, Stochastic Processes and their Applications 128 (2018), no. 1, 255 -290.

[5] C. R. Bacon, Practical portfolio performance measurement and attribution, The Wiley Finance Series, Wiley, 2005.

[6] E.J. Baurdoux, Z. Palmowski, and M.R. Pistorius, On future drawdowns of Lévy processes, Stochastic Processes and their Applications 127 (2017), no. 8, 2679 -2698.

[7] J. Bertoin, Lévy processes, Cambridge University Press, 1996.

[8] X. Chen, D. Landriault, B. Li, and D. Li, On minimizing drawdown risks of lifetime investments, Insurance: Mathematics and Economics 65 (2015), no. C, 46-54.

[9] J. Cvitanic and I. Karatzas, On portfolio optimization under "drawdown" constraints, Constraints, ima lecture notes in mathematics \& applications 65, 1994, pp. 77-88.

[10] A. Dassios and S. Wu, Parisian ruin with exponential claims, London School of Economics and Political Science, LSE Library, 2008.

[11] A. Dassios and S. Wu, On barrier strategy dividends with Parisian implementation delay for classical surplus processes, Insurance Math. Econom. 45 (2009), no. 2, 195-202.

[12] S. J. Grossman and Z. Zhou, Optimal investment strategies for controlling drawdowns, Mathematical Finance 3 (1993), no. 3, 241-276.

[13] A. E. Kyprianou, Fluctuations of Lévy processes with applications - Introductory lectures, Second, Universitext, Springer, Heidelberg, 2014.

[14] D. Landriault, B. Li, and $\mathrm{H}$. Zhang, On magnitude, asymptotics and duration of drawdowns for Lévy models, Bernoulli 23 (201702), no. 1, 432-458.

[15] D. Landriault, J.-F. Renaud, and X. Zhou, Occupation times of spectrally negative Lévy processes with applications, Stochastic Process. Appl. 121 (2011), no. 11, 2629-2641.

[16] J. P. Lehoczky, Formulas for stopped diffusion processes with stopping times based on the maximum, Ann. Probab. 5 (197708), no. 4, 601-607.

[17] B. Li, G. E. Willmot, and J. T. Y. Wong, A temporal approach to the Parisian risk model, J. Appl. Probab. 55 (2018), no. 1, 302-317. 
[18] M. A. Lkabous, I. Czarna, and J.-F. Renaud, Parisian ruin for a refracted Lévy process, Insurance Math. Econom. 74 (2017), 153-163.

[19] R. Loeffen, Z. Palmowski, and B.A. Surya, Discounted penalty function at parisian ruin for Lévy insurance risk process, Insurance: Mathematics and Economics 83 (2018), 190-197.

[20] R. L. Loeffen, I. Czarna, and Z. Palmowski, Parisian ruin probability for spectrally negative Lévy processes, Bernoulli 19 (2013), no. 2, 599-609.

[21] R. L. Loeffen, J.-F. Renaud, and X. Zhou, Occupation times of intervals until first passage times for spectrally negative Lévy processes, Stochastic Process. Appl. 124 (2014), no. 3, 1408-1435.

[22] A. Mijatović and M. R. Pistorius, On the drawdown of completely asymmetric Lévy processes, Stochastic Processes and their Applications 122 (2012), no. 11, 3812 -3836.

[23] Z. Palmowski and J. Tumilewicz, Pricing insurance drawdown-type contracts with underlying Lévy assets, Insurance: Mathematics and Economics 79 (2018), 1 -14.

[24] L. Pospisil and J. Vecer, Portfolio sensitivity to changes in the maximum and the maximum drawdown, Quantitative Finance 10 (2010), no. 6, 617-627.

[25] J.-L. Pérez and K. Yamazaki, Mixed periodic-classical barrier strategies for Lévy risk processes, Risks 6 (2018Apr), no. 2, 33.

[26] F. Schuhmacher and M. Eling, Sufficient conditions for expected utility to imply drawdown-based performance rankings, Journal of Banking \& Finance 35 (2011), no. 9, 2311-2318.

[27] L. Shepp and A. N. Shiryaev, The Russian Option: Reduced Regret, The Annals of Applied Probability 3 (1993), no. 3, $631-640$.

[28] B. A. Surya, Parisian excursion below a fixed level from the last record maximum of Lévy insurance risk process (2019), 311-326.

[29] H. M. Taylor, A Stopped Brownian Motion Formula, The Annals of Probability 3 (1975), no. 2, 234 -246.

[30] C Yin and KC Yuen, Exact joint laws associated with spectrally negative Lévy processes and applications to insurance risk theory, Frontiers of Mathematics in China 9 (2014), $1453-1471$.

\section{Appendix A. Proofs}

A.1. Proof of Lemma 3. First, we consider the Gerber-Shiu distribution of $Y$ at the Poisson observation $T_{a}^{+}$, that is

$$
\mathbb{E}_{x}^{y}\left[\mathrm{e}^{-q T_{a}^{+}} \mathbf{1}_{\left\{Y_{T_{a}^{+}}-a \in \mathrm{d} z, T_{a}^{+}<\infty\right\}}\right] .
$$

To do so, we first rewrite 22 as

$$
\mathbb{E}_{x}^{y}\left[\mathrm{e}^{-q T_{a}^{+}-p\left(Y_{T_{a}^{+}}-a\right)}\right]=\frac{\lambda}{\psi_{q+\lambda}(p)}\left(\frac{Z_{q}\left(a-s, \Phi_{\lambda+q}\right)}{Z_{q}^{\prime}\left(a, \Phi_{\lambda+q}\right)} Z_{q}^{\prime}(a, p)-Z_{q}(a-s, p)\right),
$$

and aim to invert $(36)$ with respect to $p$. From $(8)$, we know that

$$
\frac{Z_{q}(a-s, p)}{\psi_{q+\lambda}(p)}=\int_{0}^{\infty} \mathrm{e}^{-p z} \mathcal{W}_{a-s}^{(q, \lambda)}(a-s+z) \mathrm{d} z
$$

Also, from (6),

$$
\frac{Z_{q}^{\prime}(a, p)}{\psi_{q+\lambda}(p)}=\frac{p Z_{q}(a, p)}{\psi_{q+\lambda}(p)}-\left(1+\frac{\lambda}{\psi_{q+\lambda}(p)}\right) W_{q}(a) .
$$

Given that from (37),

$$
p \frac{Z_{q}(a, p)}{\psi_{q+\lambda}(p)}-W_{q}(a)=\int_{0}^{\infty} \mathrm{e}^{-p z} \mathcal{W}_{a}^{(q, \lambda) \prime}(a+z) \mathrm{d} z,
$$

we obtain

$$
\frac{Z_{q}^{\prime}(a, p)}{\psi_{q+\lambda}(p)}=\int_{0}^{\infty} \mathrm{e}^{-p z} \mathcal{W}_{a}^{(q, \lambda) \prime}(a+z) \mathrm{d} z-\lambda W_{q}(a) \int_{0}^{\infty} \mathrm{e}^{-p z} W_{q+\lambda}(z) \mathrm{d} z .
$$


Substituting (37) and (39) into (36), and inverting the resulting equation with respect to $p$ yield (24).

Using the Gerber-Shiu distribution in $(24)$, we obtain

$$
\begin{aligned}
& \mathbb{E}_{x}^{y}\left[\mathrm{e}^{-q T_{a}^{+}} W_{p}\left(b-Y_{T_{a}^{+}}\right) \mathbf{1}_{\left\{T_{a}^{+}<\infty\right\}}\right] \\
= & \int_{0}^{b-a} W_{p}(b-a-z) \mathbb{E}_{x}^{y}\left[\mathrm{e}^{-q T_{a}^{+}} \mathbf{1}_{\left\{Y_{T_{a}^{+}}-a \in \mathrm{d} z, T_{a}^{+}<\infty\right\}}\right] \\
= & \lambda \frac{Z_{q}\left(a-s, \Phi_{\lambda+q}\right)}{Z_{q}^{\prime}\left(a, \Phi_{\lambda+q}\right)} \int_{0}^{b-a} W_{p}(b-a-z)\left(\mathcal{W}_{a}^{(q, \lambda) \prime}(a+z)-\lambda W_{q}(a) W_{q+\lambda}(z)\right) \mathrm{d} z \\
& -\lambda \int_{0}^{b-a} W_{p}(b-a-z) \mathcal{W}_{a-s}^{(q, \lambda)}(a-s+z) \mathrm{d} z,
\end{aligned}
$$

which completes the proof of (25). Finally, the proof of 26 follows similarly.

A.2. Proof of Theorem 6. First, we set $x=0$. For $a<y<b$, performing a standard probabilistic decomposition using the strong Markov property of $Y$ and spectral negativity of $X$, we can write

$$
\mathbb{E}^{y}\left[\mathrm{e}^{-q \kappa_{a, b}^{\lambda, r}}\right]=\mathbb{E}^{y}\left[\mathrm{e}^{-q\left(r \wedge \rho_{b}^{+}\right)} \mathbf{1}_{\left\{\rho_{a}^{-}>r \wedge \rho_{b}^{+}\right\}}\right]-\mathbb{E}^{y}\left[\mathrm{e}^{-q \rho_{a}^{-}} \mathbf{1}_{\left\{\rho_{a}^{-} \leq r \wedge \rho_{b}^{+}\right\}}\right] \mathbb{E}^{a}\left[\mathrm{e}^{-q \kappa_{a, b}^{\lambda, r}}\right] .
$$

For $y \leq a$ and using again the strong Markov property of $Y$, we have

$$
\mathbb{E}^{y}\left[\mathrm{e}^{-q \kappa_{a, b}^{\lambda, r}}\right]=\mathbb{E}^{y}\left[\mathrm{e}^{-q T_{a}^{+}} \mathbb{E}^{Y_{a}^{+}}\left[\mathrm{e}^{-q \kappa_{a, b}^{\lambda, r}} \mathbf{1}_{\left\{\kappa_{a}^{\lambda, r}<\infty\right\}}\right] \mathbf{1}_{\left\{T_{a}^{+}<\infty\right\}}\right] .
$$

Substituting (40) into (41), yields

$$
\begin{aligned}
\mathbb{E}^{y}\left[\mathrm{e}^{-q \kappa_{a, b}^{\lambda, r}}\right]=\mathbb{E}^{y}\left[\mathrm{e}^{-q T_{a}^{+}}\right. & \left.\mathbb{E}^{Y_{T_{a}^{+}}}\left[\mathrm{e}^{-q\left(r \wedge \rho_{b}^{+}\right)} \mathbf{1}_{\left\{\rho_{a}^{-}>r \wedge \rho_{b}^{+}\right\}}\right] \mathbf{1}_{\left\{T_{a}^{+}<\infty\right\}}\right] \\
+ & \mathbb{E}^{y}\left[\mathrm{e}^{-q T_{a}^{+}} \mathbb{E}^{Y T_{a}^{+}}\left[\mathrm{e}^{-q \rho_{a}^{-}} \mathbf{1}_{\left\{\rho_{a}^{-} \leq r \wedge \rho_{b}^{+}\right\}}\right] \mathbf{1}_{\left\{T_{a}^{+}<\infty\right\}}\right] \mathbb{E}^{a}\left[\mathrm{e}^{-q \kappa_{a}^{\lambda, r}}\right],
\end{aligned}
$$

for all $y \in \mathbb{R}$. From the definitions of $g_{\lambda}^{(q)}$ and $h_{\lambda}^{(q)}$, we can now rewrite 42 as

$$
\mathbb{E}^{y}\left[\mathrm{e}^{-q \kappa_{a, b}^{\lambda, r}}\right]=h_{\lambda}^{(q)}(a, b, r, y)+g_{\lambda}^{(q)}(a, b, r, y) \mathbb{E}^{a}\left[\mathrm{e}^{-q \kappa_{a, b}^{\lambda, r}}\right] .
$$

Let $y=a$. Solving $(43)$ for $\mathbb{E}^{a}\left[\mathrm{e}^{-q \kappa_{a, b}^{\lambda, r}} \mathbf{1}_{\left\{\kappa_{a}^{\lambda, r}<\tau_{b}^{+}\right\}}\right]$, one obtains

$$
\mathbb{E}^{a}\left[\mathrm{e}^{-q \kappa_{a, b}^{\lambda, r}} \mathbf{1}_{\left\{\kappa_{a, b}^{\lambda, r}<\tau_{b}^{+}\right\}}\right]=\frac{h_{\lambda}^{(q)}(a, b, r, a)}{1-g_{\lambda}^{(q)}(a, b, r, a)} .
$$

Thus, plugging 440 in (43), we obtain

$$
\mathbb{E}^{y}\left[\mathrm{e}^{-q\left(\kappa_{a, b}^{\lambda, r}-r\right)}\right]=h_{\lambda}^{(q)}(a, b, r, y)+\frac{g_{\lambda}^{(q)}(a, b, r, y)}{1-g_{\lambda}^{(q)}(a, b, r, a)} h_{\lambda}^{(q)}(a, b, r, a) .
$$


Taking the Laplace transform (in $r$ ) of $g_{\lambda}^{(q)}$ and $h_{\lambda}^{(q)}$ and using Fubini's theorem together with 20) and 21, we obtain

$$
\begin{aligned}
\int_{0}^{\infty} \mathrm{e}^{-\theta r} g_{\lambda}^{(q)}(a, b, r, y) \mathrm{d} r & =\frac{\mathbb{E}^{y}\left[\mathrm{e}^{-q T_{a}^{+}} \mathbb{E}^{Y_{T_{a}^{+}}}\left[\mathrm{e}^{-q \rho_{a}^{-}} \mathbf{1}_{\left\{\rho_{a}^{-} \leq \mathrm{e}_{\theta} \wedge \rho_{b}^{+}\right\}}\right] \mathbf{1}_{\left\{T_{a}^{+}<\infty\right\}}\right]}{\theta} \\
& =\frac{1}{\theta} \frac{1}{W_{\theta+q}(b-a)} \mathbb{E}^{y}\left[\mathrm{e}^{-q T_{a}^{+}} W_{\theta+q}\left(b-Y_{T_{a}^{+}}\right) \mathbf{1}_{\left\{T_{a}^{+}<\infty\right\}}\right],
\end{aligned}
$$

and

$$
\begin{aligned}
& \int_{0}^{\infty} \mathrm{e}^{-\theta r} h_{\lambda}^{(q)}(a, b, r, y) \mathrm{d} r \\
= & \frac{\mathbb{E}^{y}\left[\mathrm{e}^{-q T_{a}^{+}} \mathbb{E}^{Y_{a}^{+}}\left[\mathrm{e}^{-(q+\theta) \rho_{b}^{+}} \mathbf{1}_{\left\{\rho_{b}^{+}<\rho_{a}^{-}\right\}}\right] \mathbf{1}_{\left.\left\{T_{a}^{+}<\infty\right\}\right]}\right.}{\theta} \\
& -\frac{\mathbb{E}^{y}\left[\mathrm { e } ^ { - q T _ { a } ^ { + } } \mathbb { E } ^ { Y _ { T _ { a } ^ { + } } } \left[\mathrm{e}^{\left.-(\theta+q)\left(\rho_{a}^{-} \wedge \rho_{b}^{+}\right)\right]} \mathbf{1}_{\left.\left\{T_{a}^{+}<\infty\right\}\right]}\right.\right.}{\theta+q}+\frac{\mathbb{E}^{y}\left[\mathrm{e}^{-q T_{a}^{+}}\right]}{\theta+q} \\
= & \frac{q}{(\theta+q) \theta} \mathbb{E}^{y}\left[\mathrm{e}^{-q T_{a}^{+}} Z_{\theta+q}\left(b-Y_{T_{a}^{+}}\right) \mathbf{1}_{\left\{T_{a}^{+}<\infty\right\}}\right]+\frac{\mathbb{E}^{y}\left[\mathrm{e}^{-q T_{a}^{+}}\right]}{\theta+q} \\
& +\frac{1}{(\theta+q) \theta} \frac{\left(\theta-q Z_{\theta+q}(b-a)\right)}{W_{\theta+q}(b-a)} \mathbb{E}^{y}\left[\mathrm{e}^{-q T_{a}^{+}} W_{\theta+q}\left(b-Y_{T_{a}^{+}}\right) \mathbf{1}_{\left\{T_{a}^{+}<\infty\right\}}\right] .
\end{aligned}
$$

The above expectations can be computed using $(25)$ and $(26)$. The final result follows by the spatial homogeneity of $X$.

A.3. Proof of Corollary 7, In this case, the probabilistic decomposition leads to

$$
\begin{aligned}
\mathbb{E}^{y}\left[\mathrm{e}^{-q \kappa_{a}^{\lambda, r}}\right]= & \mathrm{e}^{-q r} \mathbb{E}^{y}\left[\mathrm{e}^{-q T_{a}^{+}}\right]-\mathrm{e}^{-q r} \mathbb{E}^{y}\left[\mathrm{e}^{-q T_{a}^{+}} \mathbb{P}^{Y} T_{a}^{+}\left(\rho_{a}^{-} \leq r\right)\right] \\
& +\mathbb{E}^{y}\left[\mathrm{e}^{-q T_{a}^{+}} \mathbb{E}^{Y_{a}^{+}}\left[\mathrm{e}^{-q \rho_{a}^{-}} \mathbf{1}_{\left\{\rho_{a}^{-} \leq r\right\}}\right] \mathbf{1}_{\left\{T_{a}^{+}<\infty\right\}}\right] \mathbb{E}^{a}\left[\mathrm{e}^{-q \kappa_{a}^{\lambda, r}}\right],
\end{aligned}
$$

for all $y \in \mathbb{R}$. The functions $g_{\lambda}^{(q)}$ and $h_{\lambda}^{(q)}$ reduce to

and

$$
g_{\lambda}^{(q)}(a, r, y)=\mathbb{E}^{y}\left[\mathrm{e}^{-q T_{a}^{+}} \mathbb{E}^{Y_{T_{a}^{+}}}\left[\mathrm{e}^{-q \rho_{a}^{-}} \mathbf{1}_{\left\{\rho_{a}^{-} \leq r\right\}}\right]\right]
$$

$$
h_{\lambda}^{(q)}(a, r, y)=\mathrm{e}^{-q r} \mathbb{E}^{y}\left[\mathrm{e}^{-q T_{a}^{+}}\right]-\mathrm{e}^{-q r} \mathbb{E}^{y}\left[\mathrm{e}^{-q T_{a}^{+}} \mathbb{P}^{Y} T_{a}^{+}\left(\rho_{a}^{-} \leq r\right)\right] .
$$

Thus, from 22 , we have

$$
\begin{aligned}
\int_{0}^{\infty} \mathrm{e}^{-\theta r} \mathbb{E}^{y}\left[\mathrm{e}^{-q T_{a}^{+}} \mathbb{P}^{Y T_{a}^{+}}\left(\rho_{a}^{-} \leq r\right)\right] \mathrm{d} r=\frac{1}{\theta} \mathbb{E}^{y}\left[\mathrm{e}^{-q T_{a}^{+}} \mathrm{e}^{\Phi_{\theta}\left(a-Y_{T_{a}^{+}}\right)}\right] \\
=\frac{\lambda}{\theta(\theta-(q+\lambda))}\left(Z_{q}^{\prime}\left(a-y, \Phi_{\theta}\right) \frac{Z_{q}\left(a-s, \Phi_{\lambda+q}\right)}{Z_{q}^{\prime}\left(a, \Phi_{\lambda+q}\right)}-Z_{q}\left(a-y, \Phi_{\theta}\right)\right),
\end{aligned}
$$

and

$$
\int_{0}^{\infty} \mathrm{e}^{-\theta r} g_{\lambda}^{(q)}(a, r, y) \mathrm{d} r=\frac{1}{\theta} \mathbb{E}^{y}\left[\mathrm{e}^{-q T_{a}^{+}} \mathrm{e}^{\Phi_{\theta+q}\left(a-Y_{T_{a}^{+}}\right)}\right]
$$




$$
=\frac{\lambda}{\theta(\lambda-\theta)}\left(Z_{q}\left(a-y, \Phi_{\theta+q}\right)-Z_{q}^{\prime}\left(a-y, \Phi_{\theta+q}\right) \frac{Z_{q}\left(a-s, \Phi_{\lambda+q}\right)}{Z_{q}^{\prime}\left(a, \Phi_{\lambda+q}\right)}\right) .
$$

Now, using the following identities (which can be proved using Kendall's identity and (14))

$$
\begin{aligned}
\frac{Z_{q}\left(u, \Phi_{q+\theta}\right)}{(\theta-\lambda) \theta} & =\int_{0}^{\infty} \mathrm{e}^{-\theta r}\left(\mathrm{e}^{\lambda r} \int_{0}^{r} \mathrm{e}^{-(\lambda+q) s} \Lambda^{(q)}(u ; s) \mathrm{d} s\right) \mathrm{d} r \\
\frac{Z_{q}\left(u, \Phi_{\theta}\right)}{(\theta-(\lambda+q)) \theta} & =\frac{\int_{0}^{\infty} \mathrm{e}^{-\theta r}\left(\int_{0}^{r}\left(q+\lambda \mathrm{e}^{(\lambda+q)(r-s)}\right) \Lambda^{(q)}(u, s) \mathrm{d} s\right) \mathrm{d} r}{\lambda+q}, \\
\frac{Z_{q}^{\prime}\left(u, \Phi_{q+\theta}\right)}{(\theta-\lambda) \theta} & =\int_{0}^{\infty} \mathrm{e}^{-\theta r}\left(\mathrm{e}^{\lambda r} \int_{0}^{r} \mathrm{e}^{-(\lambda+q) s} \Lambda^{(q)^{\prime}}(u, s) \mathrm{d} s\right) \mathrm{d} r \\
\frac{Z_{q}^{\prime}\left(u, \Phi_{\theta}\right)}{(\theta-\lambda-q) \theta} & =\frac{\int_{0}^{\infty} \mathrm{e}^{-\theta r}\left(\int_{0}^{r}\left(q+\lambda \mathrm{e}^{(\lambda+q)(r-s)}\right) \Lambda^{(q)^{\prime}}(u, s) \mathrm{d} s\right) \mathrm{d} r}{\lambda+q},
\end{aligned}
$$

leads to

$$
\begin{aligned}
g_{\lambda}^{(q)}(a, r, y)=\frac{\lambda}{\lambda+q} \frac{Z_{q}\left(a-y, \Phi_{\lambda+q}\right)}{Z_{q}^{\prime}\left(a, \Phi_{\lambda+q}\right)}\left(\int_{0}^{r}\left(q+\lambda \mathrm{e}^{(\lambda+q)(r-s)}\right) \Lambda^{(q) \prime}(a, s) \mathrm{d} s\right) \\
-\frac{\lambda}{\lambda+q} \int_{0}^{r}\left(q+\lambda \mathrm{e}^{(\lambda+q)(r-s)}\right) \Lambda^{(q)}(a-y, s) \mathrm{d} s,
\end{aligned}
$$

and

$$
\begin{aligned}
h_{\lambda}^{(q)}(a, r, y)=\frac{\lambda \mathrm{e}^{-q r}}{\lambda+q} & \left(Z_{q}(a-s)-\frac{Z_{q}\left(a-s, \Phi_{\lambda+q}\right)}{Z_{q}^{\prime}\left(a, \Phi_{\lambda+q}\right)} Z_{q}^{\prime}(a)\right) \\
+ & \frac{Z_{q}\left(a-y, \Phi_{\lambda+q}\right)}{Z_{q}^{\prime}\left(a, \Phi_{\lambda+q}\right)}\left(\lambda \mathrm{e}^{\lambda r} \int_{0}^{r} \mathrm{e}^{-(\lambda+q) s} \Lambda^{(q)^{\prime}}(a, s) \mathrm{d} s\right) \\
& -\lambda \mathrm{e}^{\lambda r} \int_{0}^{r} \mathrm{e}^{-(\lambda+q) s} \Lambda^{(q)}(a-y, s) \mathrm{d} s .
\end{aligned}
$$

For $y=a$, we obtain

$$
h_{\lambda}^{(q)}(a, r, a)=\frac{\lambda \mathrm{e}^{\lambda r}}{\lambda+q}-\frac{\lambda \mathrm{e}^{-q r}}{\lambda+q} \frac{\int_{0}^{r}\left(q+\lambda \mathrm{e}^{(\lambda+q)(r-s)}\right) \Lambda^{(q) \prime}(a, s) \mathrm{d} s}{Z_{q}^{\prime}\left(a, \Phi_{\lambda+q}\right)}-\frac{\lambda \mathrm{e}^{-q r}}{\lambda+q} \frac{q W_{q}(a)}{Z_{q}^{\prime}\left(a, \Phi_{\lambda+q}\right)},
$$

and

$$
1-g_{\lambda}^{(q)}(a, r, y)=\mathrm{e}^{\lambda r}-\frac{\left(\lambda \mathrm{e}^{\lambda r} \int_{0}^{r} \mathrm{e}^{-(\lambda+q) s} \Lambda^{(q)^{\prime}}(a, s) \mathrm{d} s\right)}{Z_{q}^{\prime}\left(a, \Phi_{\lambda+q}\right)} .
$$

Thus, we have the following expression for $\mathbb{E}^{a}\left[\mathrm{e}^{-q \kappa_{a}^{\lambda, r}} \mathbf{1}_{\left\{\kappa_{a}^{\lambda, r}<\tau_{b}^{+}\right\}}\right]$.

$$
\begin{aligned}
& \mathbb{E}^{a}\left[\mathrm{e}^{-q \kappa_{a}^{\lambda, r}} \mathbf{1}_{\left.\left\{\kappa_{a}^{\lambda, r}<\tau_{b}^{+}\right\}\right]}\right.=\frac{\lambda}{\lambda+q}\left(1-\frac{q\left(\int_{0}^{r} \Lambda^{(q) \prime}(u, s) \mathrm{d} s+W_{q}(a)\right)}{\mathrm{e}^{(\lambda+q) r}\left(Z_{q}^{\prime}\left(a, \Phi_{\lambda+q}\right)-\lambda \int_{0}^{r} \mathrm{e}^{-(\lambda+q) s} \Lambda^{(q)^{\prime}}(a, s) \mathrm{d} s\right)}\right) \\
&=\frac{\lambda}{\lambda+q}\left(1-\frac{\mathrm{e}^{-q r} \mathcal{D}^{(q) \prime}(a, r)}{\mathcal{K}^{(q) \prime}(a, r, \lambda)}\right) . \\
& 14
\end{aligned}
$$


The result follows by substituting the above formula and the expressions of $g_{\lambda}^{(q)}(a, r, y)$ and $h_{\lambda}^{(q)}(a, r, y)$ into 47 .

\section{APPENDIX B.}

Landriault et al. [14] studied the Laplace transform of $\kappa^{r}$ and provided two expressions depending on whether the Lévy process has bounded or unbounded variation. We summarize their results in the following theorem.

Theorem 12. Let $q \geq 0$ and $r>0$.

If $X$ is of unbounded variation,

$$
\mathbb{E}^{0}\left[\mathrm{e}^{-q\left(\kappa^{r}-r\right)}\right]=\frac{\mathrm{e}^{-q r} \int_{r}^{\infty} \frac{1}{s} p_{s}^{X}(0) \mathrm{d} s}{q \int_{0}^{r} \mathrm{e}^{-q t} \int_{t}^{\infty} \frac{1}{s} p_{s}^{X}(0) \mathrm{d} s \mathrm{~d} t+\mathrm{e}^{-q r} \int_{r}^{\infty} \frac{1}{s} p_{s}^{X}(0) \mathrm{d} s},
$$

where $p_{s}^{X}(z)$ is the density of $X_{s}$.

If $X$ is of bounded variation,

$$
\mathbb{E}^{0}\left[\mathrm{e}^{-q\left(\kappa^{r}-r\right)}\right]=\frac{\int_{0}^{\infty} \mathbb{P}\left(\bar{X}_{r} \leq y\right) \Pi(-\mathrm{d} y)}{q+\int_{0}^{\infty} \mathbb{P}\left(\bar{X}_{\mathrm{e}_{q} \wedge r} \leq y\right) \Pi(-\mathrm{d} y)} .
$$

where $\mathrm{e}_{q}$ is an independent exponential rv with mean $1 / q$.

Now, we make a connection between the results in [14] and [28]. Using (13), we have

$$
\begin{aligned}
& \mathcal{D}^{(q)}(0, r)=1+q \int_{0}^{r} \Lambda^{(q)}(0, s) \mathrm{d} s=\mathrm{e}^{q r}, \\
& \mathcal{D}^{(q)^{\prime}}(u, r)=q W_{q}(0)+q \int_{0}^{r} \Lambda^{(q)^{\prime}}(0, s) \mathrm{d} s .
\end{aligned}
$$

Hence, we obtain

$$
\begin{aligned}
\mathbb{E}^{0}\left[\mathrm{e}^{-q \kappa^{r}}\right] & =\mathrm{e}^{-q r}\left(D^{(q)}(0, r)-\frac{\Lambda^{(q)}(0, r)}{\Lambda^{(q) \prime}(0, r)} D^{(q) \prime}(0, r)\right) \\
& =1-q \frac{W_{q}(0)+\int_{0}^{r} \Lambda^{(q) \prime}(0, s) \mathrm{d} s}{\Lambda^{(q) \prime}(0, r)} .
\end{aligned}
$$

Using Kendall's identity (15), Tonelli's theorem and an integration by parts, we obtain the following Laplace transforms

$$
\int_{0}^{\infty} \mathrm{e}^{-\theta r} \Lambda^{(q) \prime}(0, r) \mathrm{d} r=-W_{q}(0)+\frac{\Phi_{\theta}}{\theta-q},
$$

and

$$
\int_{0}^{\infty} \mathrm{e}^{-\theta r}\left(\int_{0}^{r} \Lambda^{(q) \prime}(0, s) \mathrm{d} s\right) \mathrm{d} r=-\frac{W_{q}(0)}{\theta}+\frac{\Phi_{\theta}}{\theta(\theta-q)} .
$$

In the case where $X$ has paths of unbounded variation $\left(W_{q}(0)=0\right)$, and by Laplace inversion, we obtain

and

$$
\Lambda^{(q) \prime}(0, r)=q \mathrm{e}^{q r} \int_{0}^{r} \mathrm{e}^{-q t} \int_{t}^{\infty} \frac{1}{s} p_{s}^{X}(0) \mathrm{d} s \mathrm{~d} t+\int_{r}^{\infty} \frac{1}{s} p_{s}^{X}(0) \mathrm{d} s
$$

$$
\int_{0}^{r} \Lambda^{(q) \prime}(0, s) \mathrm{d} s=\mathrm{e}^{q r} \int_{0}^{r} \mathrm{e}^{-q t} \int_{t}^{\infty} \frac{1}{s} p_{s}^{X}(0) \mathrm{d} s \mathrm{~d} t .
$$


Putting the pieces together, we obtain the result in 50

Now, we suppose that $X$ is of bounded variation $\left(W_{q}(0)>0\right)$. First, we notice from 53 and (54) that

$$
\Lambda^{\prime}(0, r)=\Lambda^{(q) \prime}(0, r)-q \int_{0}^{r} \Lambda^{(q) \prime}(0, s) \mathrm{d} s-q W_{q}(0) .
$$

Then, we can rewrite 52 as follows

$$
\mathbb{E}^{0}\left[\mathrm{e}^{-q \kappa^{r}}\right]=\mathrm{e}^{-q r} \frac{\Lambda^{\prime}(0, r)}{\Lambda^{(q) \prime}(0, r)} .
$$

Finally, one can prove that

$$
\Lambda^{\prime}(0, r)=W(0) \int_{0}^{\infty} \mathbb{P}\left(\bar{X}_{r} \leq y\right) \Pi(-\mathrm{d} y),
$$

and

$$
\mathrm{e}^{-q r} \int_{0}^{r} \Lambda^{(q) \prime}(0, s) \mathrm{d} s=W_{q}(0) \int_{0}^{\infty} \mathbb{P}\left(\bar{X}_{\mathrm{e}_{q} \wedge r} \leq y\right) \Pi(-\mathrm{d} y),
$$

by showing that the Laplace transforms in $r$ of both sides are equal. Hence, we showed that

$$
\mathbb{E}^{0}\left[\mathrm{e}^{-q\left(\kappa^{r}-r\right)}\right]=\frac{\Lambda^{\prime}(0, r)}{\Lambda^{(q) \prime}(0, r)},
$$

which corresponds to 35 .

Department of Statistics and Actuarial Science, University of Waterloo, Waterloo, On, N2L 3G1, CANADA

E-mail address: dlandria@uwaterloo.ca

Department of Statistics and Actuarial Science, University of Waterloo, Waterloo, On, N2L 3G1, CANADA

E-mail address: bin.li@uwaterloo.ca

School of Mathematical Sciences, University of Southampron, Highfield, Southampton, SO17 1BJ, United Kingdom

E-mail address: m.a.1kabous@soton.ac.uk 